\title{
Hacia una Nueva Propuesta de Evaluación del Éxito Educativo
}

\section{Towards a New Proposal to Assess Educational Success}

\author{
Pau López-Vicente * \\ Irene Cussó-Parcerisas \\ Eva Rodríguez-Garcia \\ Jordi Riera-Romaní \\ Universitat Ramon Llull
}

\begin{abstract}
Solemos utilizar el concepto de éxito como antagónico de fracaso considerando solamente indicadores de fácil cuantificación, con la escasa autocrítica al reduccionismo que supone esta opción. Entonces, nos preguntamos ¿qué significa tener éxito escolar? El artículo pretende profundizar en la comprensión de éste fenómeno en nuestro contexto. En primer lugar, se analizan cuáles son los fines comunes de la educación expresados en los preámbulos de las diversas leyes de educación desarrolladas en el período democrático en nuestro país desde la Constitución española de 1978. En segundo lugar, centrándonos en trabajos de investigación sobre esta temática en nuestro territorio, identificamos las diferentes representaciones de los conceptos de éxito y fracaso escolar y cómo se llega a un criterio de medida para valorarlos. A partir de este doble análisis se pone de relieve que mayoritariamente los indicadores que se manejan para evaluar el éxito o el fracaso escolar se centran preferentemente en las calificaciones académicas o evaluaciones estandarizadas de diagnóstico de competencias, dejando en segundo término el resto de fines expresados en las leyes de educación, como pueden ser la adquisición de otras calidades personales y sociales. Por esta razón, concluimos el artículo con una propuesta metodológica para el análisis del fenómeno que enfatiza una aproximación multimétodo, para encontrar evidencias de los elementos-causa en términos de indicadores - que contribuyen al éxito educativo teniendo en cuenta todos los objetivos explícitos en las leyes de educación.
\end{abstract}

Descriptores: Éxito escolar, Fracaso escolar, PISA, Evaluación.

Often educational success is understood as opposed to the concept of school failure. Usually quantifiable indicators are used to measure the phenomena, with little criticism of the reductionism this option poses. In this article we query about how we could assess educational success from a wider perspective in our context. First of all, this study analyses the common principles that appear in the forewords of the different Spanish Education Laws that have been in force during the democratic period in our country since 1978. Secondly, through several research articles about this subject, we identify the different conceptualizations and approaches to measure the phenomena in our territory. The analysis highlights that educational success or failure is mainly measured through indicators that use academic grades or standardized diagnostic tests. Our main finding is that there is a preference to link school success and failure to academic achievement, whereas other goals expressed in the Educational Laws are considered secondary. Therefore, we conclude the article with a methodological proposal to assess educational success from a multimethod approach that takes into account the different objectives explicit in the Educational Laws.

Keywords: School success, School failure, PISA, Assessment.

*Contacto: paulv@blanquerna.url.edu

ISSN: $1696-4713$

www.rinace.net/reice/
Recibido: $\quad 1$ de septiembre 2015

$1^{\text {a }}$ Evaluación: 17 de diciembre 2015

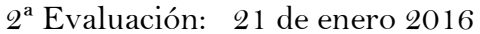

Aceptado: $\quad 2$ de febrero 2016 
Proyecto subvencionado por el Ministerio de Economía y Competitividad, España. Número de referencia del proyecto: EDU2012-39497-c04-O1

\section{Introducción}

Actualmente el fracaso escolar sigue siendo uno de los conceptos que despiertan más interés en medios de comunicación y en todos los entornos relacionados con la educación. A nivel de discurso público, parecen tener un mayor impacto los enunciados con una visión pesimista de la realidad, pero ¿no sería más pertinente centramos en las fortalezas? Por ejemplo: ¿Cómo valoramos el éxito escolar en nuestro país? ¿Qué logros constatamos?

Se habla indistintamente de éxito educativo, éxito académico y éxito escolar, y lo mismo ocurre con el término fracaso pero hay dificultad para interpretar dichos fenómenos. Además, se utiliza el concepto de éxito como antagónico de fracaso considerando solamente indicadores de fácil cuantificación, con escasa autocrítica al reduccionismo que supone esta opción. Entonces, ¿qué significa tener éxito escolar? ¿Se trata simplemente de aprobar los exámenes?, o bien ¿ise considera como principal evidencia incuestionable el hecho de conseguir un título oficial de estudios? Observamos que se utilizan los resultados en las pruebas PISA para determinar quiénes tienen fracaso o éxito. Por otro lado, no se puede reducir todo únicamente a las calificaciones académicas, dado que el éxito se relaciona también con la adquisición de otras cualidades personales y sociales muy explícitas en las leyes de educación.

Constatando que los conceptos que se manejan de éxito y de fracaso presentan significados difusos, queremos profundizar en su comprensión analizando cómo se utilizan en nuestro contexto. Tres son los objetivos específicos de nuestro estudio:

- Comprender cuál es la misión y los objetivos generales de la educación expresados en las leyes de educación de nuestro país en el período democrático.

- Analizar la interpretación del fenómeno del éxito/fracaso escolar en trabajos de investigación publicados en la última década en revistas españolas con factor de impacto.

- Proponer una alternativa a la interpretación y medida del fenómeno del éxito/fracaso escolar.

En primer lugar, y para aproximarnos a estos conceptos, analizaremos los fines de la educación descritos en las diferentes leyes de educación desarrolladas a partir de la Constitución española de 1978, para descubrir los puntos comunes y las coincidencias entre ellas que enmarcan lo que se considera como éxito educativo.

Seguidamente, y centrándonos en los trabajos de investigación publicados en revistas españolas con factor de impacto, analizamos las diferentes representaciones de los conceptos de éxito y fracaso escolar y qué criterios e indicadores de medida se utilizan para valorarlos, contrastando estas representaciones con los principios comunes que han orientado las leyes de educación. A partir de ésta reflexión crítica entorno a los indicadores de evaluación del éxito educativo de acuerdo con los objetivos generales de la educación, presentamos y concluimos con una nueva propuesta para evaluar el éxito/fracaso escolar que se ajuste a la misión y objetivos generales de la educación. 


\section{Fundamentación Teórica}

A través de la Convención sobre los Derechos del Niño (CDN) en 1989 los estados miembros reconocen el derecho de los niños a la educación. Concretamente, en el artículo 29 se define este derecho como el desarrollo de la personalidad, el talento y la capacidad mental y física de los niños/as; asimismo, el conocimiento y respecto de los derechos humanos y libertades fundamentales, el respeto de su propia identidad cultural y familiar y de los valores de su entorno, así como la tolerancia y el espíritu de comprensión hacia otras identidades sociales y culturales. Es a partir de estos principios que los estados deben establecer normas y criterios específicos en cada contexto para garantizar el derecho a la educación.

En el caso de España y desde la aprobación de la Constitución española en 1978, se han redactado y aplicado diversas leyes de educación. Enmendando la última ley preconstitucional de 1970 Ley General de Educación (LGE, 1970) aparece la primera ley del periodo constitucional en 1985, la Ley Reguladora del Derecho a la Educación (LRDE 1985), y sucesivamente la Ley Orgánica General del Sistema Educativo (LOGSE, 1990), la Ley Orgánica de Calidad de la Educación (LOCE, 2002), la Ley Orgánica de Educación (LOE, 2006) y la Ley Orgánica de Mejora de la Calidad de la Educación (LOMCE, 2013). Con la alternancia en el poder de los dos grandes partidos políticos del estado español (Partido Popular (PP) y Partido Socialista Obrero Español (PSOE)), se han redactado nuevas leyes de educación con las huellas específicas de cada color político y con el soporte de otros partidos minoritarios, pero sin llegar al consenso en ninguna ocasión (Bolívar, 2004). La mayoría parlamentaria de uno u otro partido no ha propiciado el debate exhaustivo para llegar a un acuerdo -a pesar de algunos intentos ${ }^{1}-\mathrm{y}$, de este modo, las leyes se han aprobado por votación mayoritaria aplicando simplemente el reglamento de la cámara. Un claro ejemplo es la polémica generada a raíz de la aprobación de la última ley, la LOMCE (2013), que en palabras de Mozos (2014) "se preocupa más de lo instrumental que de lo fundamental" (Mozos, 2014, p.23).

La aplicación de cada nueva ley de educación ha originado múltiples estudios comparativos y análisis centrados en una preocupación omnipresente: el fracaso escolar. Sin una intencionalidad muy explícita, se ha llevado a cabo un proceso de valoración permanente para intentar medir el grado de eficacia o de fracaso de cada nueva ley y de las normativas correspondientes. Los resultados siempre han mostrado datos de España con déficits importantes, comparándolos con los de la media europea, y así se ha justificado la reforma de una ley, la aparición de normativas específicas en relación a contenidos y horarios docentes, o la formulación de nuevas leyes con el propósito explícito de alcanzar mayores éxitos. En el preámbulo o presentación de cada nueva ley, se explicitan las diferencias que ofrece con respecto a la anterior y sus bondades para intentar una mejora de resultados en la educación.

Analizaremos en primer lugar, las coincidencias entre los fines u objetivos de las diversas leyes, desde la LRDE (1985) hasta la LOMCE (2013). ¿Qué fines u objetivos se

1 El 27 de enero de 2010, Ángel Gabilondo, Ministro de Educación del gobierno socialista presenta el documento "Propuestas para un pacto social y político por la educación", con los objetivos de la educación en España para la década 2010-2020 y la formulación de 124 propuestas. El principal partido de la oposición, el Partido Popular, rechazó la propuesta y no se llegó a ningún acuerdo. 
mantienen a lo largo de tres décadas? Las evidencias cualitativas o cuantitativas en relación a la consecución de estos objetivos deberían indicar el grado de éxito o fracaso.

En la declaración de principios que orientan cada ley, en su introducción y/o preámbulo, constatamos seis objetivos que consideramos claramente coincidentes:

Primero: Rendimiento en los aprendizajes académicos.

Consideramos dentro de esta categoría todo lo relativo al aprendizaje de los contenidos de las asignaturas así como a las capacidades estratégicas y actitudes personales para hacerles frente. Para ejemplificar esta categoría, transcribimos algunas citas de las diversas leyes: "La adquisición de hábitos intelectuales y técnicas de trabajo, así como de conocimientos científicos, técnicos, humanísticos, históricos y estéticos. (LRDE, art. 2c; LOGSE, art. 1, 1c, art. 18 y 19). En la LOGSE se especifica "el desarrollo de las capacidades creativas y del espíritu crítico"; la LOE (art. 22) añade "el desarrollo de hábitos saludables, el ejercicio físico y el deporte” y la LOCE (art. 1, i) y la LOE (art. 2, f y art. 23, g) "el desarrollo de la capacidad de los alumnos para regular su propio aprendizaje, confiar en sus aptitudes y conocimientos, así como para desarrollar la creatividad, la iniciativa personal y el espíritu emprendedor".

Segundo: Capacidades profesionales, aprendizajes laborales y sociales.

Dentro de esta categoría incluimos los objetivos que mencionan de forma preferente la capacitación laboral y la participación activa en la sociedad.

Destacamos algunas citas textuales: "La capacitación para el ejercicio de actividades profesionales" (LRDE, art. 2d; LOGSE, art. 18; LOE, art. 22) y "la preparación para participar activamente en la vida social y cultural" (LRDE, art. 2, f; LOGSE, art. 18 y art. 19, e, h, j). En la LOE (art. 2, k) se enfatiza "la preparación para el ejercicio de la ciudadanía y para la participación activa en la vida económica, social y cultural, con actitud crítica y responsable y con capacidad de adaptación a las situaciones cambiantes de la sociedad del conocimiento".

Tercero: El desarrollo integral de la persona.

Dentro de esta categoría consideramos los objetivos que hacen referencia explícita al desarrollo global de la persona, integrando conocimientos, valores y personalidad del individuo.

Para ejemplificar esta categoría, destacamos algunos fragmentos textuales: "El pleno desarrollo de la personalidad del alumno" (LRDE, art.2, a; LOGSE, art. 1, 1a y art. 19, d, i; LOCE, art.1, a; LOE, art. 2, a; LOMCE, art. 1, b). Entrando más en detalle, la LOGSE (art. 2, 3a) especificaba "la formación personalizada, que propicie una educación integral en conocimientos, destrezas y valores morales de los alumnos en todos los ámbitos de la vida, personal, familiar, social y profesional. En la LOE (art. 2, a) se expresa "el pleno desarrollo de la personalidad y de las capacidades de los alumnos" y la LOMCE (art. 1, b) matiza "el pleno desarrollo de la personalidad a través de la educación, la inclusión educativa”.

Cuarto: Actitudes pro-sociales, valores y exigencias de una buena ciudadanía.

Consideramos dentro de esta categoría los objetivos que explicitan el desarrollo de actitudes que favorecen el entendimiento y el respeto entre las personas así como las actitudes básicas para la buena convivencia. 
Coincide en diversas leyes "la formación en el respeto de los derechos y libertades fundamentales y en el ejercicio de la tolerancia y de la libertad dentro de los principios democráticos de convivencia” (LRDE, art.2, b, g; LOGSE, art. 1, 1b y art. 19, d, i; LOCE, art. 22, 2; LOE, art. 2, c y art. 23, a, d). En la LOGSE (art. 2, 3c) se añade "la efectiva igualdad de derechos entre los sexos, el rechazo a todo tipo de discriminación, y el respeto a todas las culturas" y "el fomento de los hábitos de comportamiento democrático”. En la LRDE (art. 2, b), la LOE (art. 2, b, c; art. 1, l) y la LOCE (art. 1, b) se explicita "la igualdad de derechos y oportunidades entre hombres y mujeres y en la igualdad de trato y no discriminación de las personas con discapacidad” y la LOMCE (art. 1, l) hace hincapié en "la prevención de la violencia de género". También en la LOE (art. 2, b, 2 y art. 23, c) y la LOMCE (art. 1, l) se aportan otros matices: "la igualdad de derechos entre los sexos, que ayuden a superar cualquier tipo de discriminación, así como la práctica de la solidaridad" y "como elemento compensador de las desigualdades personales, culturales, económicas y sociales”.

Abundando en este objetivo de la convivencia se hace hincapié en "la formación para la paz, la cooperación y la solidaridad entre los pueblos” (LRDE, art. 2, g; LOGSE, art.1, 8). La LRDE (art. 2, g), la LOE (art. 1, k) y la LOMCE (art. 1, K) añaden "para la prevención de conflictos y para la resolución pacífica de los mismos”. La LRDE (art. 2, g) y la LOMCE (art. 1, k) especifican "en todos los ámbitos de la vida personal, familiar y social” y la LOMCE (art. 1, k) concreta "en especial en el acoso escolar". La LOE (art. 2, e) añade "el respeto a los derechos humanos, la vida en común, la cohesión social, la cooperación y solidaridad entre los pueblos” y la LOGSE (art. 2, 3k) y la LOE (art. 23, k) "la adquisición de valores que propicien el respeto hacia los seres vivos y el medio ambiente".

\section{Quinto: Educación permanente.}

Dentro de esta categoría encontramos los objetivos que mencionan la necesidad de la formación a lo largo de toda la vida.

Como ejemplificación, transcribimos una cita que se repite en la LOGSE y en la LOCE: "El principio básico de la educación permanente [...] a lo largo de toda la vida" (LOGSE, art. 2, 1; LOCE, art. 1, e).

\section{Sexto: Respeto a la diversidad.}

En esta categoría incluimos los objetivos que enfatizan el respeto a la pluralidad cultural.

Encontramos ejemplos textuales en la LRDE, la LOGSE y la LOE: "La formación en el respeto de la pluralidad lingüística y cultural de España” (LRDE, art. 2, c; LOGSE, art. 1, e; LOE, art. 2, j y art. 23, h) así como "la capacitación para la comunicación en la lengua oficial y cooficial, si la hubiere, y en una o más lenguas extranjeras".

En relación a estas seis categorías u objetivos que podemos considerar que se han mantenido a lo largo de tres décadas, ¿de qué indicadores y datos disponemos para valorarlos y determinar el grado de éxito o de fracaso de las leyes? ¿Qué objetivos se valoran realmente y qué indicadores se utilizan en los estudios realizados sobre éxito o fracaso escolar?

A continuación, se hará un análisis de investigaciones entorno el fenómeno del éxito/fracaso. 


\section{Método}

El propósito es analizar cómo se ha interpretado y definido el fenómeno del éxito/fracaso escolar en trabajos de investigación publicados en la última década en revistas españolas de impacto. Realizamos un análisis descriptivo que nos permitirá responder a la pregunta sobre cuáles son las conceptualizaciones prevalentes en la investigación científica entorno a este fenómeno.

Primero se presentan los criterios para la selección de las revistas y los artículos así como el proceso de clasificación y análisis. Seguidamente, se comentan las conceptualizaciones predominantes halladas en relación a las categorías comunes que orientan las leyes de educación y se ponen de relieve los indicadores o variables más utilizados en las investigaciones para medir el fenómeno.

\subsection{Criterios para la selección de la muestra de artículos}

Desde una perspectiva hermenéutico-interpretativa, queremos comprender qué definiciones encontramos de éxito/fracaso escolar en la investigación científica de la última década. Ante la imposibilidad de abarcar todos los trabajos publicados en este período, restringimos nuestro ámbito de análisis, orientando nuestro estudio a partir de unos criterios de búsqueda y selección de artículos específicos.

Los artículos seleccionados han sido publicados entre el año 2001 y junio de 2014 y se han incluido artículos de investigación, ya sean de carácter exploratorio, descriptivo o con voluntad explicativa, que usan tanto metodologías cuantitativas como cualitativas. El objeto de estudio de los artículos seleccionados es el fracaso o el éxito escolar en la etapa secundaria obligatoria. Se han incluido también artículos que investigan las transiciones entre la etapa primaria y la etapa secundaria, así como entre la etapa secundaria obligatoria y formación postobligatoria. Las investigaciones seleccionadas están contextualizadas en el estado español pero también se han seleccionado estudios de comparativa internacional que incluyeran España. Cómo último criterio, cabe mencionar que solamente se analizan los artículos publicados en revistas españolas de impacto, según el índice Journal Citation Reports 2012 (JCR) ISI Web of Knowledge en las siguientes temáticas: Psychology, Education E Educational Research, Sociology.

Finalmente, se han encontrado 53 artículos entre el año 2001 y junio de 2014 entorno al éxito o fracaso escolar en ocho de las diez revistas españolas con factor de impacto $J C R$ 2012 y correspondientes a las temáticas citadas. No se han encontrado artículos en este período y teniendo en cuenta los criterios de selección en las revista de impacto según JCR 2012: Infancia y Aprendizaje ni en la Revista Enseñanza de las Ciencias. Concretamente, cabe precisar que en la primera revista en períodos anteriores al estudiado sí hay algunos artículos en relación a esta temática. La segunda revista es una publicación más focalizada en la didáctica de las ciencias y los artículos no tenían relación con el tema de estudio. De estos, solo 41 cumplen también los otros criterios mencionados anteriormente. Número de artículos seleccionados y analizados de las diferentes revistas con factor de impacto: Revista de Psicodidáctica (1), Revista Española de Pedagogía (1), Revista de Educación (31), Reis-Revista Española de Investigaciones Sociológicas (1), Revista Internacional de Sociología (1), Revista Cultura y Educación (2), Revista ESE. Estudios sobre educación (2) y Revista Educación XX1 (2). 
En relación a la temática de los artículos, 16 se relacionan con el tema del éxito. Consideramos aquí todos los que hablan de éxito, logros académicos, rendimiento académico, promoción de curso o eficacia escolar. Los otros 25 se refieren al tema del fracaso escolar. Dentro de ésta categoría también incluimos los que hablan del fenómeno de la repetición de curso, el abandono escolar temprano o el absentismo.

\subsection{Proceso de categorización de los artículos}

Para la categorización de los artículos se han clasificado en relación a las categorías encontradas con los principios que orientan las leyes de educación. El análisis ha sido de carácter interpretativo, primero a nivel individual y, en una segunda fase contrastando y triangulando los resultados a nivel grupal.

Sobre el discurso y definición del fenómeno se observan artículos que definen el éxito/fracaso escolar en relación a:

- Rendimiento académico (categoría $1^{\mathrm{a}}$ ), incluimos aquí también los artículos que hablan de obtención de la graduación o titulación obligatoria.

- Capacidades profesionales, aprendizajes laborales y sociales, así como la formación permanente (categorías $2^{\mathrm{a}}$ y $5^{\mathrm{a}}$ ).

- Desarrollo integral de la persona (categoría $3^{\mathrm{a}}$ ).

- Actitudes pro-sociales, valores y exigencias de una buena ciudadanía (categorías $4^{\mathrm{a}}$ y $6^{\mathrm{a}}$ ).

De este modo, se han reducido a 4 las 6 categorías comunes que orientan las leyes de educación.

En relación a las variables e indicadores empleados para medir el éxito o fracaso escolar también se han identificado tres tendencias que clasificamos de la siguiente manera:

- Niveles de competencia a través de PISA u otras pruebas estandarizadas de competencias.

- Calificaciones (notas).

- Estudio de trayectorias y transiciones académicas.

\section{Resultados}

En este apartado presentamos el resultado a partir del análisis interpretativo-descriptivo de los artículos y sus conceptualizaciones predominantes en correspondencia con los objetivos comunes de las leyes de educación. Asignamos los 41 artículos en las 4 categorías descritas anteriormente, en función de sus definiciones entorno al éxito/fracaso escolar. Sin embargo, no son categorías excluyentes y un artículo puede situarse simultáneamente en más de una categoría. El gráfico 1 muestra el resultado de la clasificación.

Se observa como la mayoría de artículos, concretamente 38, definen el tema del éxito/fracaso en relación al rendimiento académico. Hemos incluido aquí también los artículos que definen el fenómeno en relación a la obtención o no de la titulación obligatoria correspondiente. Entendemos aquí la graduación como una forma de 
rendimiento académico, ya que para llegar a esta meta el rendimiento tiene un peso determinante (aunque no siempre sea el único criterio) y es un requisito necesario para la superación de etapas. El resto de categorías se encuentran menos representadas en los artículos: capacidades profesionales y actitudes pro-sociales, ambas en 5 artículos y el desarrollo integral incluido en 4 artículos.

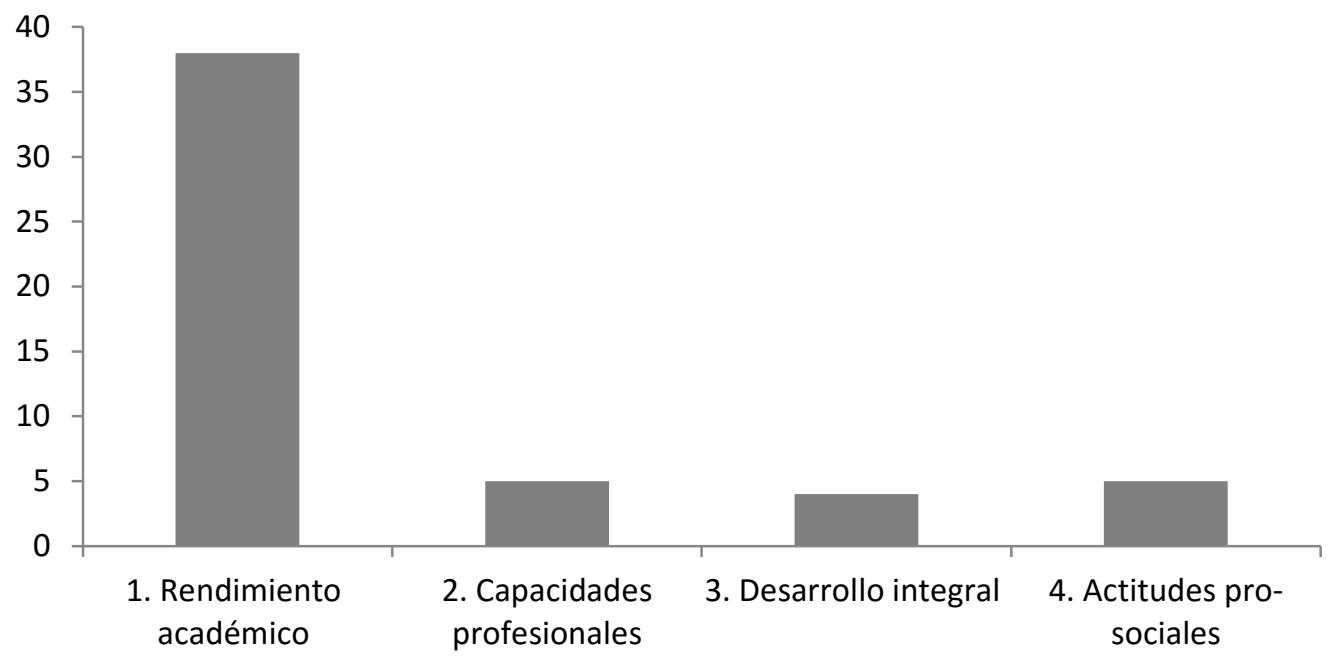

Gráfico 1. Número de artículos en función de las categorías establecidas a partir del análisis de los principios comunes de las leyes de educación

Fuente: Elaboración propia.

A pesar de esa menor consideración es importante poner de relieve la emergencia de estas representaciones en los artículos porque implica que cuando hablamos de éxito/fracaso escolar no se trata de un concepto limitado al ámbito académico, sino que también se habla de capacidades de la persona en relación a sí misma y al ámbito profesional y social.

A continuación, focalizamos la atención en las variables, indicadores y perspectivas metodológicas empleadas para medir el éxito/fracaso en las investigaciones analizadas. Identificamos en los artículos tres tendencias mayoritarias según la propuesta de medición observada: el 32\% (13 artículos de 41) se basan en evaluaciones estandarizadas de diagnóstico de competencias (programa PISA u otras pruebas); el 22\% (9 artículos de 41) toman las calificaciones académicas; y, finalmente, el 36\% (15 artículos de 41) corresponden a estudios sobre trayectorias y transiciones académicas. Los artículos pueden pertenecer a más de una categoría en caso de utilizar diferentes métodos y en ésta clasificación hemos desestimado los artículos de carácter teórico o sin propuesta de indicadores para el tema de estudio (Ayala, Molina y Prieto, 2012; Cobacho y Pons, 2006; Escudero y Martínez, 2012; Poy, 2009; Pozo, Suárez y García-Cano, 2012; Vega y Aramendi, 2010).

El 32\% de los artículos se aproximan al fenómeno del éxito/fracaso escolar a partir de la explotación de los resultados obtenidos en el programa PISA en sus diversas ediciones o utilizan otras pruebas o evaluaciones estandarizadas de diagnóstico de competencias. El programa PISA evalúa cada 3 años el nivel de competencia de los alumnos de 15 años a escala internacional, centrando su atención en las siguientes áreas temáticas: lectura, matemáticas y resolución de problemas y ciencias. Cada edición se centra en una de las 
áreas. Concretamente, entre los artículos analizados encontramos un artículo basado en los resultados PISA-2003 (Ruiz, 2009); cuatro artículos que utilizan los resultados de la edición PISA-2006 (Calero, Chois y Waisgrais, 2010; Gil, 2012; Roca, 2010; Salinas y Santín, 2012); dos artículos con datos de PISA-2009 (Choi y Calero, 2013; Cordero, Manchón y Simancas, 2014). Y, finalmente, tres investigaciones que cuentan con los resultados de diferentes ediciones (Cordero, Crespo y Pedraja, 2013; Guio y Choi, 2014; Nieto y Recamán, 2012). Los siguientes estudios utilizan otras pruebas u evaluaciones estandarizadas de diagnóstico de competencias (Flecha, García, Gómez y Latorre, 2009; Gil, 2011; Goikoetxea, 2007).

En estas investigaciones se define el éxito/fracaso como el rendimiento obtenido a través de estas pruebas, es decir, el indicador de éxito/fracaso es el nivel de competencia alcanzado en la realización de estas evaluaciones. En el caso de las pruebas PISA se considera una posición situación de riesgo de fracaso cuando no se alcanza el nivel dos de rendimiento en las competencias. Aunque, tal como advierten los mismos autores, el riesgo de fracaso entendido de esta forma no corresponde con las cifras del llamado "fracaso escolar administrativo" (Choi y Calero, 2013, p. 570). Por ello, es necesaria una profunda reflexión sobre las formas de medir el fracaso o el éxito considerando todas las dimensiones implicadas.

La ventaja de utilizar estas pruebas es, por un lado, la obtención de datos relativos al alumno y su contexto escolar y familiar (Cordero, Manchón y Simancas, 2014). Por otro lado, ofrece una comparativa entre los diferentes países analizados, así como una visión sistemática del panorama educativo cada tres años y, por lo tanto, puede ser útil para configurar las políticas e inversiones educativas en cada país (Cordero, Crespo y Pedraja, 2013, UNESCO, 2015).

Sin embargo, creemos que esta aproximación limita la concepción de éxito o fracaso, ya que se centra únicamente en los resultados obtenidos del nivel competencial en relación a ciertas disciplinas. El siguiente fragmento pone de relieve ésta apreciación: "el output educativo se restringe a los resultados obtenidos en los test, sin prestar atención a otros resultados del proceso educativo, como los aspectos no cognitivos" (Cordero, Crespo y Pedraja, 2011, p. 290). Ciertamente, en la última edición de la evaluación PISA 2012, se introducen otras dimensiones de análisis como es la resolución creativa de problemas o el nivel de competencia financiera, relacionada con la toma de decisiones en la vida cotidiana pero no necesariamente relacionada con la competencia matemática $(\mathrm{OECD}$, 2014 a y b). Se dejan de lado, no obstante, otros objetivos de la educación como son las capacidades profesionales, el desarrollo integral y los valores sociales.

Encontramos un segundo grupo de artículos, el 22\%, que recogen las calificaciones académicas como indicador de éxito/fracaso. Las notas recogidas a través de los expedientes académicos o bien a través de un registro subjetivo se convierten en una fuente de información válida para medir el logro académico (Bernardi y Cebolla, 2014; Mena, Fernández y Rivière, 2010; Rodríguez, Fita y Torrado, 2004). La interpretación se puede realizar de distintas maneras. Las notas organizadas a partir de una escala facilitan una lectura cualitativa del logro: insuficiente, bajo, medio, alto (Barca, Peralba, Porto, Marcos y Brenlla, 2011) o bien más cuantitativa: número de materias suspendidas (Broc, 2006). Hay una tendencia en observar las notas obtenidas en disciplinas concretas, sobretodo, matemáticas y lenguas (4 de las 9 investigaciones) (Nieves-Pérez, Betancort y Cabrera, 2013; Santos, Godás y Lorenzo, 2012; Servicio de inspección educativa de 
Madrid-Capital, 2002 y 2003). Otras investigaciones utilizan las notas de conocimiento del medio y ciencias naturales (Santos, Godás y Lorenzo, 2012); y literatura (Santos, Godás y Lorenzo, 2012; Servicio de inspección educativa de Madrid-Capital, 2002 y 2003). Esta última observación destaca que las áreas instrumentales son las más valoradas en relación con el éxito/fracaso escolar, quedando al margen las valoraciones en materias como la educación musical, la educación visual-plástica, la educación física, la filosofía o el pensamiento humanístico-social, disciplinas, entre otras, que contribuyen al desarrollo integral de la persona.

La tercera y última perspectiva encontrada en los artículos es la comprensión del éxito/fracaso a partir del estudio de las transiciones entre las etapas educativas y del análisis de las trayectorias académicas. Se interpreta aquí éxito o bien fracaso en función de la certificación o no de los títulos (obligatorios o postobligatorios) y la consiguiente decisión de continuar o abandonar los estudios. Agrupamos en esta categoría 15 artículos (el 36\%), aunque a continuación los clasificamos en función de la aproximación que desarrollan: cuantitativa, cualitativa o mixta.

En primer lugar, 6 de los 15 artículos elaboran su análisis a partir de datos procedentes de encuestas, como por ejemplo, Transición, la Educación, la Formación e Inserción Laboral (ETEFIL) en su edición de 2005 elaborada por el Instituto Nacional de Estadística (INE) y el Ministerio de Educación (MEC) (Gracia, Casal, Merino y Sánchez, 2013); Spanish Labour Force Survey en sus ediciones anuales desde 2005 a 2008 (SLF) (Bernardi y Requena, 2010); micro datos de la Encuesta de Población Activa del $2^{\circ}$ trimestre de 2007 (Fernández, Muñoz, Braña y Antón, 2010); base de datos de la Organización para la Cooperación y el Desarrollo Económicos -OECD en sus siglas en inglés-Education at a Glance 2007 y Ministerio de Educación o la recogida de datos a partir de elaboración de cuestionarios específicos sobre el abandono en población adolescente (Mora, 2010); micro datos procedentes de la Encuesta de Población Activa del INE (Casquero y Navarro, 2010) o el análisis del fenómeno a partir de técnicas estadísticas de análisis de datos, como análisis de regresión (Alegre y Benito, 2010).

En segundo lugar, encontramos investigaciones que para comprender el fenómeno utilizan el método biográfico. Esta aproximación cualitativa identificada en 4 de los 15 artículos se basa en el relato de las historias de vida narradas por los propios alumnos, complementado a veces con entrevistas con otros agentes clave del entorno, observaciones de carácter más etnográfico y recogida y análisis de documentación relacionada con los casos de estudio (Adame y Salvà, 2010; Calderón, 2014; Martínez, 2001; Mena, Fernández y Rivière, 2010). Permite profundizar en las experiencias de fracaso y abandono desde el punto de vista de los propios alumnos.

Por último, observamos en 5 de los 15 artículos que aplican una doble aproximación. Por un lado, cuentan con datos estadísticos y complementan su análisis con una metodología cualitativa que les permite profundizar en el fenómeno del éxito/fracaso escolar, a partir de entrevistas y observaciones (Aramendi, Vega y Buján, 2012; Fernández, 2011; Flecha, García, Gómez, Latorre, 2009; García, 2005; Serra y Palaudàries, 2010). Se trata de investigaciones que apuestan por una aproximación multimétodo.

En síntesis, hemos observado diferentes formas de medir e interpretar el alcance del fenómeno del éxito/fracaso escolar. Constatamos una tendencia a centrarse en la evaluación de los resultados a partir del rendimiento desarrollado en ciertas áreas. Por lo tanto, a determinar el éxito/fracaso, en función del nivel obtenido en la evaluación de 
competencias PISA o tomando las calificaciones académicas (lo que representa un 54\%). Existe, sin embargo, la emergencia de otras maneras de representar el fracaso/éxito desde un enfoque más comprensivo teniendo en cuenta los relatos de los alumnos, sus trayectorias y transiciones, lo que permite investigar mejor los mecanismos que llevan al éxito/fracaso y evaluarlo en las otras dimensiones: profesional, desarrollo integral y actitudes y valores pro-sociales.

A continuación, se presenta una alternativa para representar el fracaso/éxito desde un enfoque considerado como multimétodo.

\section{Propuesta para la Evaluación del Éxito Educativo desde un Enfoque Multimétodo}

Teniendo en cuenta las categorías construidas a partir de nuestro análisis de los fines expresados en las diversas leyes de educación, destacamos cuatro objetivos o ámbitos que deberían ser objeto de análisis y evaluación para obtener evidencias en relación al éxito o al fracaso educativo, y poder, de este modo, determinar las modificaciones pertinentes.

- Rendimiento académico.

- Capacidades profesionales.

- Desarrollo integral.

- Prosocialidad.

Para obtener evidencias en relación al éxito o al fracaso, apostamos por un sistema de análisis y evaluación que vaya en la línea de investigaciones con un enfoque más biográfico, relatando las historias de vida de los propios alumnos y complementándolo con entrevistas a sus profesores y otros agentes educativos, tal como proponen Adame y Salvà (2010), Calderón (2014), Martínez (2001) y Mena, Fernández y Rivière (2010). O bien, ofreciendo un análisis con doble aproximación: por un lado los datos estadísticos y por otro, datos analizados con una metodología cualitativa que permitan profundizar sobre el fenómeno a través de entrevistas o cuestionarios, tal como plantean Aramendi, Vega y Buján (2012), Fernández (2011), Flecha, García, Gómez y Latorre (2009), García (2005) y Serra y Palaudàries (2010).

Para llevar a cabo todo el proceso, se precisaría, en una primera fase, de una comisión especializada formada por reconocidos expertos de distintos ámbitos (a nivel estatal y autonómico) incluyendo profesorado de educación primaria y secundaria, con amplio recorrido en la docencia, la tutoría y la planificación educativa. Se trataría de revisar las propuestas actuales en relación al rendimiento académico y de acordar los objetivos prioritarios de los tres ámbitos restantes, así como los sistemas específicos para hacer efectiva su evaluación.

En una segunda fase este círculo central tendría el apoyo de un segundo círculo más amplio, con expertos representantes de todos los territorios, que ejercerían una función de contraste con las propuestas y conclusiones de la comisión central. 
La tercera y última fase sería la aplicación de las distintas pruebas a cargo de los responsables pedagógicos de los centros seleccionados, con el apoyo de equipos expertos miembros del segundo círculo de contraste.

\subsection{Sistemas y estrategias de evaluación del éxito educativo}

En estas pruebas, se partiría de una muestra significativa de centros de diversos contextos socioculturales utilizando la categorización del Instituto IDEA (Marchesi y Martín, 2002) y se aplicaría a los cursos de $5^{\circ}$ de EP y de $3^{\circ}$ de ESO. De esta manera, los resultados permitirían implementar medidas de corrección específicas en $6^{\circ}$ de EP y en $4^{\circ}$ de ESO, si fuera preciso.

Se evaluarían aspectos significativos de cada ámbito, obteniendo datos cuantitativos y cualitativos a través de técnicas específicas que concretamos a continuación.

\section{a) Rendimiento académico}

El primer ámbito se refiere al aprendizaje de los contenidos de las asignaturas y a las capacidades estratégicas y actitudes personales para hacerles frente. Observamos la estrecha relación de este primer aspecto con la perspectiva del Informe Delors (1996), concretamente cuando menciona e insiste en la necesidad de pasar de la noción de calificación a la noción de competencia.

Técnicas: Pruebas estandarizadas, tipo PISA, referidas a todas las áreas, incluyendo la formación artística, la educación musical y la educación física.

\section{b) Capacidades profesionales}

Éste ámbito se relaciona con las capacidades laborales y sociales y con la formación permanente que también se reivindica en el informe Delors. En este sentido se concibe la formación en sentido amplio. Es una acción que se desarrolla a lo largo de nuestra vida y en cualquier parte gracias a las tecnologías de la información y la comunicación que lo permiten. Lo que se ha denominado Life Long Learning y Ubiquitous Learning Environments, respectivamente. Para afrontar este modelo de aprendizaje es necesario superar la concepción que "sólo aprendemos en la escuela y durante nuestra escolarización” (Civís y Riera, 2007, p.15). Además, en la sociedad actual debido a la enorme cantidad de fuentes de información, el conocimiento tiene un carácter 'líquido', utilizando la metáfora de Zigmund Bauman, en el sentido que es flexible y cambiante. En este contexto incierto se requiere de un aprendizaje continuo para desarrollarse plenamente como profesionales y ciudadanos y, siguiendo el mismo autor, además de renovar las habilidades técnicas y la educación centrada en el trabajo, también es necesario y urgente renovar la educación para crear ciudadanos (Bauman, 2007).

Técnicas: Pruebas de destrezas manipulativas, informáticas, de búsqueda de información, de organización de equipos, de improvisación ante situaciones imprevistas, de aprovechamiento de recursos, de creatividad y de perseverancia, entre otras.

\section{c) Desarrollo integral}

Este ámbito hace referencia explícita al desarrollo global de la persona, integrando conocimientos, valores y personalidad del individuo.

Tomando como referencia las cuatro esferas interseccionadas para el estudio del desarrollo, con referencia a los ámbitos físico, emocional, social y cognitivo (López, 2009) se analizarían características clave situadas en las diversas zonas de confluencia. 
Entre otras, podemos mencionar las habilidades físicas, la autoestima-autoimagen, las emociones básicas (Damasio, 2007; Punset y Bisquerra, 2015), la satisfacción en las relaciones sociales dentro de los diversos microsistemas (Bronfenbrenner, 2002) y la comprensión de situaciones o resolución de problemas (aspectos cognitivos no académicos).

Técnicas: Cuestionarios para los alumnos relacionados con los cuatro ámbitos; Entrevistas individuales para profundizar en las respuestas cuantitativas; Informes de los tutores en relación al desarrollo de los cuatro ámbitos; Informes médicos para tener en cuenta problemáticas significativas de salud, en los casos que se pudieran presentar; Informes de la familia a través de entrevistas para captar la perspectiva más específica del entorno vital más cercano.

\section{d) Prosocialidad}

Finalmente, el cuarto ámbito se centra en los valores y actitudes que favorecen el entendimiento y el respeto entre las personas para la buena convivencia. También relacionamos este cuarto apartado con Delors (1996) y más específicamente con "la ética del género humano: enseñar la ciudadanía terrestre" que aparece como uno de los siete saberes necesarios para la educación del futuro de Morin (2003).

Técnicas: Tomando como base la metodología de discusión de dilemas para el estudio del desarrollo moral (Kohlberg, 2002) se utilizarían tres técnicas: Cuestionarios ad hoc; Entrevistas; Grupos de discusión.

\section{Discusión y Conclusiones}

Para finalizar concluimos con algunas reflexiones que afloran a partir del análisis realizado. Ya desde el inicio tropezamos con tres conceptos ambiguos que, aun teniendo significados parecidos, no se pueden considerar como estrictamente sinónimos. Se habla indistintamente de éxito o fracaso educativo, académico y escolar. Es un primer escollo difícil de salvar. Cuando se hace referencia al "éxito educativo", por ejemplo, se incluyen aspectos más amplios, más allá de calificaciones académicas o de promoción de etapa. En cambio, el éxito o fracaso académico o escolar se relaciona más directamente con los resultados académicos tradicionales centrados preferentemente en las calificaciones obtenidas en las diversas asignaturas.

Las leyes de educación consideran diferentes aspectos o parámetros para expresar lo que deberían ser los fines de la educación. En los preámbulos y/o introducciones de las leyes de los últimos 40 años, se pueden constatar unas coincidencias esenciales, al menos en seis aspectos o categorías. La categoría que ocupa más espacio en los textos de los preámbulos se refiere al desarrollo integral de la persona y a las actitudes y valores prosociales (categorías 3 y 4). En este sentido, el sistema educativo español concuerda con las recomendaciones recientes de la Comisión Europea (2013) en relación a la necesidad de promover un desarrollo emocional, social, cognitivo y físico en la infancia para garantizar la igualdad de oportunidades, así como realizar un seguimiento y evaluación de los resultados educativos para la prevención del fracaso y abandono escolar de la infancia más vulnerable. De hecho, desde las leyes de educación se establece un currículum escolar que debería dar respuesta a los fines de la educación. Sin embargo, tal como señala Bolívar (2008), estos conocimientos adquiridos pueden estar demasiado desconectados de las competencias y habilidades requeridas en un mundo complejo y 
cambiante, ya que se aleja de propuestas como las definidas en $D e S e C o$, Definición y Selección de Competencias clave (Rychen y Salganik, 2005) o en el Education for All Global Monitoring Report 2012 (UNESCO, 2012), las cuales coinciden en conceptualizar competencias clave para fomentar la autonomía y bienestar del individuo que permitan su desarrollo personal, así como comunicarse, interactuar y participar en la sociedad.

Siendo rigurosos, cualquier evaluación del éxito o fracaso debería basarse en el grado de consecución de los objetivos explícitos en las leyes de educación. Sin embargo, en el análisis realizado encontramos que los indicadores que se manejan para dicha evaluación se centran preferentemente en la primera categoría (el rendimiento académico), dejando muy en segundo término o totalmente de lado el resto de fines (categorías: capacidades profesionales, desarrollo integral de la persona, valores y actitudes pro-sociales) expresados en las diversas leyes. Los estudios analizados sobre éxito/fracaso escolar se centran preferentemente en el rendimiento en áreas instrumentales como lengua y matemáticas. En algunos casos también se han incluido las ciencias sociales y naturales, pero ni la música, ni la expresión artística, ni la educación física, ni las actividades de tutoría, por citar cuatro ejemplos, han sido objeto de valoración para determinar el fracaso o el éxito educativo. De esta constatación emerge una preocupación que también enfatiza la UNESCO (2015) en su último informe sobre la educación, en el que se pone de relieve que la generalización del uso de pruebas estandarizadas para la evaluación puede reducir la calidad y relevancia de las experiencias educativas, al homogenizar los currículos.

Para cambiar la perspectiva, en este artículo hemos planteado una propuesta metodológica alternativa de análisis del fenómeno para encontrar evidencias de los elementos-causa que contribuyen al éxito educativo teniendo en cuenta todos los objetivos explícitos en las leyes de educación. Se trataría de poner el énfasis en qué deberíamos potenciar en vez de qué deberíamos evitar para favorecer el éxito. Para ello habría que determinar indicadores para todos los objetivos y averiguar el grado de logro en relación a las cuatro categorías: rendimiento académico, capacidades profesionales, desarrollo integral y prosocialidad. Para llevar a cabo todo el proceso, se debería contar con diversos grupos de expertos para elaborar y validar los indicadores (fase 1 y 2 ) y con responsables pedagógicos de los centros para aplicar la evaluación (fase 3), tal como se detalla en el apartado 5 .

En este sentido, avanzar hacia una aproximación multimétodo a partir de cuestionarios y pruebas estandarizadas aplicados a muestras significativas de la población, acompañados de entrevistas y grupos de discusión con estudiantes y con profesorado de secundaria, ofrecería una visión más amplia del fenómeno desde una doble perspectiva cuantitativa y cualitativa, al mismo tiempo que podría dar pistas mucho más fiables de lo que comporta el fracaso y el éxito educativo, así como las medidas que progresivamente se podrían implementar para la mejora.

\section{Referencias}

Adame, M.T. y Salvà, F. (2010). Abandono escolar prematuro y transición a la vida activa en una economía turística: el caso de las Baleares. Revista de Educación, 351, 185-210.

Alegre, M.A. y Benito, R. (2010). Los factores del abandono educativo temprano: España en el marco europeo. Revista de Educación, número extraordinario, 65-92. 
Aramendi, P., Vega A. y Buján, K. (2012). Los programas de diversificación curricular y los programas de cualificación profesional inicial ¿una alternativa al fracaso escolar? Revista Española de Pedagogía, 252, 237-251.

Ayala, C., Molina, V. y Prieto, R. (2012). Los compromisos singulares entre administración y centros para el éxito educativo. Revista de Educación, número extraordinario, 195-219.

Barca, A., Peralba, M., Porto, A., Marcos, J.L. y Brenlla, J.C. (2011). Metas académicas del alumnado de Educación Secundaria Obligatoria (ESO) y Bachillerato con alto y bajo rendimiento escolar. Revista de Educación, 354, 341-368.

Bauman, Z. (2007). Los retos de la educación en la modernidad líquida. Barcelona: Gedisa.

Bernardi, F. y Cebolla, H. (2014). Clase social de origen y rendimiento escolar como predictores de las trayectorias educativas. Reis - Revista Española de Investigaciones Sociológicas, 146, 322. doi $10.5477 /$ cis/reis. 146.3

Bernardi, F. y Requena, M. (2010). Inequality in educational transitions: The case of postcompulsory education in Spain. Revista de Educación, número extraordinario, 93-118.

Bolívar, A. (2004). La educación secundaria obligatoria en España: en la búsqueda de una inestable identidad. REICE. Revista Iberoamericana sobre Calidad, Eficacia y Cambio en Educación, 2(1), 1-22.

Bolívar, A. (2008). El discurso de las competencias en España: educación básica y educación superior. Revista de Docencia Universitaria, 6(2), 1-23.

Broc, M.A. (2006). Motivación y rendimiento académico en alumnos de Educación Secundaria Obligatoria y Bachillerato LOGSE. Revista de Educación, 340, 379-414.

Bronfenbrenner, U. (2002). La ecología del desarrollo humano. Barcelona: Paidós.

Calderón, I. (2014). Sin suerte pero guerrero hasta la muerte: pobreza y fracaso escolar en una historia de vida. Revista de Educación, 363, 174-193.

Calero, J., Chois, K. y Waisgrais, S. (2010). Determinantes del riesgo de fracaso escolar en España: una aproximación a través de un análisis logístico multinivel aplicado a PISA2006. Revista de Educación, número extraordinario, 225-256.

Casquero, A. y Navarro, M.L. (2010). Determinantes del abandono escolar temprano en España: un análisis por género. Revista de Educación, número extraordinario, 191-223.

Choi, A. y Calero, J. (2013). Determinantes del riesgo de fracaso escolar en España en PISA-2009 y propuestas de reforma. Revista de Educación, 362, 562-593.

Civís, M. y J. Riera. (2007). La nueva pedagogía comunitaria. Un marco renovado para la acción sociopedagógica interprofesional. Valencia: Nau llibres.

Cobacho, F. y Pons, J. (2006). Jóvenes en desventaja y cohesión social: Educación y futuro para todos. Revista de Educación, 341, 237-255.

Comisión Europea. (2013). Recomendación de la comisión, de 20 de febrero de 2013, Invertir en la infancia: romper el ciclo de las desventajas (2013/112/UE), Diario Oficial de la Unión Europea, 59, 5-16.

Cordero, J.M., Crespo, E. y Pedraja, F. (2013). Rendimiento educativo y determinantes según PISA: Una revisión de la literatura en España. Revista de Educación, 362, 273-297.

Cordero, J.M., Manchón, C. y Simancas, R. (2014). La repetición de curso y sus factores condicionantes en España. Revista de Educación, 365, 12-37. 
Delors, J. (1996). La educación encierra un tesoro. Informe a la UNESCO de la Comisión Internacional sobre la educación para el siglo XXI, presidida por Jacques Delors. Madrid: Santillana.

Damasio, A. (2007). En busca de Spinoza. Neurobiología de la emoción y los sentimientos. Barcelona: Crítica.

Escudero, J.M. y Martínez, B. (2012). Las políticas de lucha contra el fracaso escolar: ¿programas especiales o cambios profundos del sistema y la educación? Revista de Educación, número extraordinario, 174-193.

Fernández, E., Muñoz, R., Braña, F.J. y Antón, J.I. (2010). Algunas apreciaciones aritméticas sobre el fracaso y el abandono escolar en España. Revista de Educación, número extraordinario, 307-324.

Fernández, J.M. (2011). A la búsqueda de elementos diferenciadores que aumentan los resultados y las expectativas de alumnos en riesgo de exclusión educativa. Revista de Educación, 355, 309-330.

Flecha, A., García, R., Gómez, A. y Latorre, A. (2009). Participación en escuelas de éxito: una investigación comunicativa del proyecto Includ-ed. Cultura y Educación, 21(2), 183-196. doi: $10.1174 / 113564009788345899$

García, M. (2005). Culturas de Enseñanza y Absentismo escolar en la enseñanza secundaria obligatoria: estudio de casos en la ciudad de Barcelona. Revista de Educación, 338, 347-374.

Gil, J. (2011). Estatus socioeconómico de las familias y resultados educativos logrados por el alumnado. Cultura y Educación, 23(1), 141-154. doi: 10.1174/113564011794728597

Gil, J. (2012). Utilización del ordenador y rendimiento académico entre los estudiantes españoles de 15 años. Revista de Educación, 357, 375-396.

Goikoetxea, J. (2007). Descripción del desarrollo organizacional y curricular de diferentes tipos de centro y etapas educativas. Revista de Psicodidáctica, 12(2), 195-220.

Gracia, M., Casal, J., Merino, R. y Sánchez, A. (2013). Itinerarios de abandono escolar y transiciones tras la Educación Secundaria Obligatoria. Revista de Educación, 361, 65-94.

Guio, J.M. y Choi, A. (2014). Evolución del riesgo de fracaso escolar en España durante la década del 2000: Análisis de los resultados de PISA con un modelo logístico de dos niveles. Estudios sobre educación, 26, 33-62.

Kohlberg, L. (2002). La educación moral según Lawrence Kohlberg 1927-1987. Barcelona: Gedisa.

López, P. (2009). Desarrollo de la personalidad y el aprendizaje en la adolescencia. Barcelona: UOC.

Marchesi, A. y Martín, E. (comp) (2002). Evaluación de la educación secundaria. Fotografía de una etapa polémica. Instituto IDEA. Madrid: ed. Fundaciín SM.

Martínez, J.M. (2001). Esos chicos malos llamados repetidores. Un estudio de caso en un centro de secundaria. Revista de Educación, 325, 235-252.

Mena, L., Fernández, M. y Rivière, J. (2010). Desenganchados de la educación: procesos, experiencias, motivaciones y estrategias del abandono y del fracaso escolar. Revista de Educación, número extraordinario, 119-145.

Ministerio de Educación (2010). Propuesta para un pacto social y político por la educación. Recuperado de http://firgoa.usc.es/drupal/node/46144

Mora, A.J. (2010). Determinantes del abandono escolar en Cataluña más allá del nivel socioeconómico de las familias. Revista de Educación, número extraordinario, 171-190. 
Morin, E. (2003). Els set coneixements necessaris per a l'educació del futur. Informe elaborat per Edgar Morin per a la UNESCO com a contribució a la reflexió internacional sobre com educar per a un futur sostenible. Barcelona: Centre Unesco de Catalunya.

Mozos, I.M. (2014). La Ley Orgánica para la mejora de la Calidad Educativa: una reforma contestada, pero de corto alcance. Revista Española de Pedagogía, 257, 23-37.

Nieto, S. y Recamán, A. (2012). Hacia una mayor comprensión global del rendimiento académico a través de las pruebas PISA: contraste de tres hipótesis a partir de unos datos empíricos. Educación XX1, 15(1), 157-178. doi: 10.5944/educxx 1.15.1.154

Nieves-Pérez, C., Betancort, M. y Cabrera, L. (2013). Influencias de la familia en el rendimiento academico. Un estudio en Canarias. Revista Internacional de Sociología, 71(1), 169-187.

OECD. (2014a). Los jóvenes de 15 años son creativos a la hora de resolver problemas, PISA in focus (38). Recuperado de http://www.mecd.gob.es/inee/PISA-in-focus.html

OECD. (2014b). ¿Saben los jóvenes de 15 años cómo gestionar dinero?, PISA in focus (41). Recuperado de http://www.mecd.gob.es/inee/PISA-in-focus.html

Poy, R. (2009). Efectos del credencialismo y las expectativas sociales sobre el abandono escolar. Revista de Educación, número extraordinario, 147-169.

Pozo, M.T., Suárez, M. y García-Cano, M. (2012). Logros educativos y diversidad en la escuela: hacia una definición desde el consenso. Revista de Educación, 358, 59-84.

Punset, E. y Bisquerra, R. (2015). Universo de emociones. Valencia: PalauGea.

Roca, E. (2010). El abandono temprano de la educación y la formación en España. Revista de Educación, número extraordinario, 31-62.

Rodríguez, S., Fita, E. y Torrado, M. (2004). El rendimiento académico en la transición secundaria-universidad. Revista de Educación, 334, 391-414.

Ruiz, C. (2009). Las escuelas eficaces: un estudio multinivel de factores explicativos del rendimiento escolar en el área de matemáticas. Revista de Educación, 348, 355-376.

Rychen, D.S. y Salganik, L.H. (2005). The definition and selection of key competencies: executive summary. Recuperado de http://www.deseco.admin.ch/bfs/deseco/en/index/02.parsys.43469.downloadList.2296. DownloadFile.tmp/2005.dskcexecutivesummary.en.pdf

Salinas, J. y Santín, D. (2012). Selección escolar y efectos de la inmigración sobre los resultados académicos españoles en pisa 2006. Revista de Educación, 358, 382-405.

Santos, M.A., Godás, A. y Lorenzo, M. (2012). El perfil del alumnado repetidor y no repetidor en una muestra de estudiantes españoles y latinoamericanos: un estudio sobre los determinantes de sus logros académicos. Estudios sobre Educación, 23, 43-62.

Serra, C y Palaudàries, J.M. (2010). Deficiencias en el seguimiento del abandono escolar y trayectorias de continuidad del alumnado de origen inmigrado. Revista de Educación, número extraordinario, 283-305.

Servicio de inspección educativa de Madrid-Capital. (2002). El rendimiento escolar de los alumnos que promocionan a $1^{\circ}$ de ESO con evaluación negativa en matemáticas y lengua castellana. Revista de Educación, 329, 393-417.

Servicio de inspección educativa de Madrid-Capital. (2003). El rendimiento escolar de los alumnos que promocionan a $3^{\circ}$ de ESO con evaluación negativa en matemáticas y lengua castellana. Revista de Educación, 330, 385-418. 
Vega, A. y Aramendi, P. (2010). Entre el fracaso y la esperanza. Necesidades formativas del alumnado de los programas de cualificación profesional inicial. Educación XX1, 13(1), 3963. doi: 10.5944/educxx 1.13.1.276

UNESCO (2012). Education for all global monitoring report 2012. youth and skills: putting education to work. París: UNESCO.

UNESCO (2015). Rethinking education: towards a global common good? París: UNESCO. 